\title{
Secondary Membranous Nephropathy Associated with Guillain-Barré Syndrome
}

\author{
Edward J. Filippone ${ }^{a, d}$ Mitul Kanzaria $^{a}$ Rodney Bell ${ }^{b} \quad$ Eric Newman $^{a, d}$ \\ John L. Farber ${ }^{\mathrm{c}}$ \\ Departments of ${ }^{a}$ Medicine, ${ }^{b}$ Neurology and ${ }^{c}$ Pathology, and ${ }^{d}$ Division of Nephrology, \\ Thomas Jefferson University, Philadelphia, Pa., USA
}

\section{Key Words}

Membranous nephropathy · Nephrotic syndrome · Guillain-Barré syndrome

\begin{abstract}
Membranous nephropathy (MN) is one of the most common causes of nephrotic syndrome (NS) in adults. It may be primary, usually mediated by IgG4 anti-phospholipase A2 autoantibodies or secondary to various other conditions. Guillain- Barré syndrome (GBS) has been associated with $\mathrm{MN}$, but a cause and effect relation has not been proven. We present a case of concurrent development of GBS and severe NS, with renal biopsy demonstrating MN. IgG4 stain was negative, indicating that most likely, the MN was secondary and probably caused by the underlying GBS.
\end{abstract}

\section{Introduction}

Membranous nephropathy (MN) is one of the most common causes of nephrotic syndrome (NS) in adults $[1,2]$. Most cases are considered idiopathic (iMN), where autoantibodies react with one or more podocyte antigens (e.g., the M-type phospholipase A2 receptor [3]) to form the subepithelial deposits characteristic of all types of MN. However, in about $25 \%$ of the cases a secondary cause can be found, including lupus, viral infections (most notably hepatitis B), cancer, and medications [4]. Sometimes, a specific relevant antigen can be detected in the subepithelial deposits. These include the hepatitis e antigen in cases related to hepatitis B [5], carcinoembryonic antigen in colon carcinoma [6], and cationic bovine serum albumin in certain pediatric cases [7]. The pathologic features of both iMN and secondary MN are otherwise similar, but subtle differences do exist. For example, in 
lupus MN there may be mesangial proliferation by light microscopy, 'full house' positivity by immunofluorescence microscopy, and mesangial electron dense deposits by electron microscopy; features not usually found in iMN [8]. In iMN, IgG4 is the most prominent subclass detected [9], whereas in secondary cases another subclass is usually found.

Guillain-Barré syndrome (GBS) is a heterogeneous group of disorders with similar clinical presentations. Typically, it is an acute, self-limited, paralyzing illness, which peaks in 2 to 4 weeks and then subsides [10]. Most cases in the USA (about 85\%) result from a reversible, immunologically mediated, peripheral nerve demyelination. This is termed acute inflammatory demyelinating polyradiculoneuropathy [11]. In other cases (about 15\%), the immunologic attack is against axons, with sparing of myelin. If just motor neurons are involved, it is called acute motor axonal neuropathy (AMAN); if sensory fibers are affected as well, the term is acute motor and sensory axonal neuropathy (ASMAN). Various autoantibodies have been identified in this syndrome as well [12].

Glomerulonephritis has been found in association with GBS [13-19]. Some patients had pathologic confirmation but only mild clinical manifestations [14]. More commonly, however, reported cases had NS, and the most common lesion was MN [15, 17-19]. It is unclear whether this results from autoantibodies against podocyte antigens as in iMN or rather against an extrinsic (to the podocyte) antigen as in secondary cases. We present a case of severe NS occurring simultaneously with severe GBS of the axonal variety. Renal biopsy revealed MN, although immunohistochemical stain for IgG4 was completely negative. This suggests that the MN was indeed secondary, perhaps to an antigen released by the primary nerve damage. We discuss this in detail in light of the current knowledge of the iMN pathophysiology.

\section{Case Presentation}

A 69-year-old man with a history of hypertension, hypothyroidism, dyslipidemia, obstructive sleep apnea, benign prostatic hypertrophy, and stroke was in his usual state of health until bilateral lower extremity edema developed rapidly over a 2-week period. He developed shortness of breath and was admitted to an outside hospital. On examination, blood pressure was $142 / 112$, pulse 69 , respirations 18 , temperature $37^{\circ} \mathrm{C}$, and oxygen saturation $96 \%$ on 2 liters oxygen by nasal cannula. There were $2+$ lower extremity edema and mild right hand weakness, but no other focal neurologic findings. A chest radiograph revealed cardiomegaly; however, no infiltrates or evidence of interstitial edema. Complete blood count was normal, as were coagulation studies and electrolytes. Creatinine was 1.4 $\mathrm{mg} / \mathrm{dl}$ and albumin $2.4 \mathrm{~g} / \mathrm{dl}$, but liver function tests were otherwise normal. 24-h urine total protein excretion was $20,144 \mathrm{mg} /$ day. Several days after admission, the patient began to complain about arm and leg numbness. Lumbar puncture showed a glucose level of 169 $\mathrm{mg} / \mathrm{dl}$, a protein level of $35 \mathrm{mg} / \mathrm{dl}, \mathrm{WBC}$ of $0 / \mu \mathrm{l}$, and $\mathrm{RBC}$ of $1 / \mu \mathrm{l}$. The weakness progressed over several days and GBS was diagnosed. He was transferred to our hospital for further evaluation.

On transfer, the patient stated his right hand weakness began about 3 weeks prior to the lower extremity edema. He described slow worsening of this weakness and progressive right leg weakness. On neurological exam, he had no cranial nerve abnormalities, and his sensory exam was normal except for decreased pin-prick sensation on the left leg up to the knee. Motor exam showed 2/5 strength in his right deltoid and 4/5 in the left, biceps 4/5 bilaterally, triceps $4 / 5$ bilaterally, wrist flexors $4 / 5$ bilaterally, interossei $3 / 5$ on the right and $4 / 5$ on the left, hip flexors $3 / 5$ on the right and $4 / 5$ on the left, knee extensors $3 / 5$ on 
the right and 4/5 on the left, and dorsiflexion 4/5 bilaterally. A Mini-Mental State Examination did not demonstrate any cognitive impairment. Reflex exam was $1+$ to $2+$ with the exception of 0 bilateral ankle reflex; there was no Babinski reflex. An electromyography showed a severe axonal and demyelinating motor and sensory polyneuropathy. The patient was treated with a 5-day course of intravenous immunoglobulin, which showed no significant improvement. Laboratory results were negative for neuromyelitis optica antibodies, rheumatoid factor, complements, antinuclear antibodies, and Lyme titers. The renal biopsy showed MN, with diffuse thickening of glomerular basement membranes with spikes on the epithelial side and interstitial fibrosis in about $20 \%$ of the cortical surface area (fig. 1). Immunofluorescence microscopy showed 2 to $3+$ granular IgG staining of glomerular capillary walls. IgG4 subclass stain was negative. IgG1, IgG2, and IgG3 subclass stains are not routinely done at our institution. Electron microscopy revealed subepithelial electron dense deposits uniformly distributed throughout the glomerular capillary walls (fig. 2). The patient received a second round of intravenous immunoglobulin, which had to be discontinued due to acute kidney injury, however. He became intermittently hypotensive and required norepinephrine for blood pressure support. A positive urine culture for Klebsiella was treated with bactrim for 5 days. Weakness progressed and he required intubation. The patient developed facial diplegia, ophthalmoplegia as well as decreased corneal reflexes, and was found to be quadriplegic. The next day, he became hypotensive and bradycardic and proceeded to polymorphic ventricular tachycardia, which responded to cardioversion. Due to persistent refractory shock, care was withdrawn and the patient subsequently expired.

\section{Discussion}

Our case presented with concurrent NS and an axonal variety of GBS, which proved fatal. The underlying renal histology was clearly MN. The complete absence of IgG4 staining suggests that this was not the chance occurrence of iMN, but rather a true secondary case.

Renal disease in the setting of GBS is not common clinically, but it has been known for many years, first described in a clinicopathologic report from 1918 [13]. In the ensuing years, renal involvement was only occasionally noted. In 1973, Rodríguez-Iturbe et al. [14] described a consecutive series of 9 patients of whom 8 had biopsy evidence of glomerulonephritis. However, the clinical manifestations were minor, with either transient hypertension, hematuria or both. That same year, 2 case reports described NS in association with GBS [15, 16]. Both showed underlying MN, and 1 characterized the subclass as IgG4. Various case reports of NS have appeared since then, and the association has extended to the subacute [20] and chronic inflammatory polyneuropathies [21-23] as well as GBS. Most commonly, the glomerular lesion is $\mathrm{MN}$, although minimal change disease has also been reported $[24,25]$.

MN is the most common cause of NS in adults, although it may present with subnephrotic proteinuria. Most cases are considered idiopathic, but about $25 \%$ have defined secondary causes. These include viruses (hepatitis B or rarely C), cancers, medications (mercury, gold, penicillamine, NSAIDs, etc.), autoimmune diseases (lupus, sarcoid, etc.), and others. Included in the latter is GBS. The issue is whether this is merely a chance association without pathogenic relationship or not.

The absence of IgG4 in our case suggests they are related. iMN is usually characterized by a strong IgG4 reaction detectable by immunofluorescence $[9,26]$. It has recently been determined that these IgG4 antibodies may react with antigens normally expressed on the podocyte cell membrane (M-type phospholipase A2 receptor) [3], or only abnormally so 
Filippone et al.: Secondary Membranous Nephropathy Associated with Guillain-Barré Syndrome

(aldose reductase [27], superoxide dismutase [27], $\alpha$-enolase [28]). The antigen-antibody complexes are then shed to form the characteristic subepithelial deposits seen on electron microscopy. In general, an IgG4 response occurs after many months of chronic antigenic exposure, such as may occur with bee keepers or parasitic infestations [29]. It remains unknown what initiates this response in iMN.

In secondary MN, other antigens extrinsic to the glomerulus appear to be involved. These include hepatitis B viral antigens, tumor-related antigens, DNA, and cationic bovine serum albumin. The antigen may first plant in the glomerulus, with in situ formation of the immune complex, or, less likely, circulating immune complexes may deposit [30]. In any case, the immunoglobulins are usually not of the IgG4 subclass. There are several potential mechanisms by which GBS and secondary MN may be pathogenically related.

Various autoantibodies have been detected in different forms of GBS. Antibodies against the ganglioside GQ1b are found in most patients with the Miller Fisher variant of acute inflammatory demyelinating polyradiculoneuropathy [31], and antibodies against GM1, GD1a, and GD1b may be found with axonal variants [11]. These antibodies are thought to arise from molecular mimicry from a preceding infectious agent such as Campylobacter jejuni. It is possible that in occasional patients, these antibodies react, also through molecular mimicry, with one or more antigens expressed on podocyte cell membranes. Alternatively, damage to peripheral nerves may result in the release of an antigen(s), which deposits in the subepithelial space. The respective antibodies then bind, resulting in in situ immune complex formation. Finally, preformed circulating immune complexes involving one or more neural antigens may deposit in the subepithelial space [30]. Irrespective of the actual mechanism, MN is secondary to the primary nerve disease. The rather acute nature of the apparent exposure would be consistent with a non-IgG4 response [29]. In the case of a true infectious cause of GBS, the infectious agent itself could similarly deposit as the inciting antigen, form in situ immune complexes, and cause MN as well.

In MN associated with GBS, the renal lesion usually appears to be self-limiting, resolving temporally along with improvement of the neuropathy. These cases would obviously not require immunosuppressive therapy for the renal lesion. Unfortunately, in cases associated with the chronic and/or relapsing neuropathies, the renal disease appears to persist as well. The optimal treatment for these cases from the renal standpoint remains unknown.

\section{References}

1 Haas M, Meehan SM, Karrison TG, Spargo BH: Changing etiologies of unexplained adult nephrotic syndrome: a comparison of renal biopsy findings from 1976-1979 and 1995-1997. Am J Kidney Dis 1997;30:621-631.

2 Swaminathan S, Leung N, Lager DJ, et al: Changing incidence of glomerular disease in Olmsted County, Minnesota: a 30-year renal biopsy study. Clin J Am Soc Nephrol 2006;1:483-487.

-3 Beck LH Jr, Bonegio RGB, Lambeau G, et al: M-type phospholipase A2 receptor as target antigen in idiopathic membranous nephropathy. N Engl J Med 2009;361:11-21.

-4 Fervenza FC, Sethi S, Specks U: Idiopathic membranous nephropathy: diagnosis and treatment. Clin J Am Soc Nephrol 2008;3:905-919.

$\$ 5$ Lai KN, Li PKT, Lui SF, et al: Membranous nephropathy related to hepatitis B virus in adults. N Engl J Med 1991;324:1457-1463.

6 Couser WG, Wagonfeld JB, Spargo BH, Lewis EJ: Glomerular deposition of tumor antigen in membranous nephropathy associated with colonic carcinoma. Am J Med 1974;57:962-970.

7 Debiec H, Lefeu F, Kemper MJ, et al: Early-childhood membranous nephropathy due to cationic bovine serum albumin. N Engl J Med 2011;364:2101-2110.

-8 Jennette JC, Iskandar SS, Dalldorf FG: Pathology differentiation between lupus and nonlupus membranous glomerulopathy. Kidney Int 1983;24:377-385.

-9 Doi T, Mayumi M, Kanatsu K: Distribution of IgG subclasses in membranous nephropathy. Clin Exp Immunol 1984;58:57-62.

$\rightarrow 10$ Ropper AH: Current concepts: the Guillain-Barre syndrome. N Engl J Med 1992;326:1130-1136. 
Filippone et al.: Secondary Membranous Nephropathy Associated with Guillain-Barré Syndrome

11 Hahn AF: Guillain-Barre syndrome. Lancet 1998;352:635-641.

12 Ogawara K, Kuwabara S, Mori M, Hattori T, Koga M, Yuki N: Axonal Guillain-Barre syndrome: relation to anti-ganglioside antibodies and Campylobacter jejuni infection in Japan. Ann Neurol 2000;48:624-631.

13 Bradford JR, Bashford E, Wilson J: Acute infective polyneuritis. QJM 1918;os-12:88-126.

14 Rodríguez-Iturbe B, García R, Rubio L, Zabala J, Moros G, Torres R: Acute glomerulonephritis in the GuillainBarré-Strohl syndrome. Report of nine cases. Ann Intern Med 1973;78:391-395.

15 Behan PO, Lowenstein LM, Stilmant M, Sax DS: Landry-Guillain-Barré-Strohl syndrome and immunecomplex nephritis. Lancet 1973;1:850-854.

16 Peters DK, Sevitt LH, Direkze M, Bayliss SG: Landry-Guillain-Barré-Strohl polyneuritis and the nephrotic syndrome. Lancet 1973;1:1183-1184.

17 Talamo TS, Borochovitz D: Membranous glomerulonephritis associated with the Guillain-Barre syndrome. Am J Clin Pathol 1982;78:563-566.

18 Murphy BF, Gonzales MF, Ebeling P: Membranous glomerulonephritis and Landry-Guillain-Barre syndrome. Am J Kidney Dis 1986;8:267-270.

19 Panjwani M, Truong LD, Eknoyan G: Membranous glomerulonephritis associated with inflammatory demyelinating peripheral neuropathies. Am J Kidney Dis 1996;27:279-283.

-20 Emsley HCA, Molloy J: Inflammatory demyelinating polyradiculoneuropathy associated with membranous glomerulonephritis and thrombocytopaenia. Clin Neurol Neurosurg 2002;105:23-26.

-21 Witte AS, Burke JF: Membranous glomerulonephritis associated with chronic progressive demyelinating neuropathy. Neurology 1987;37:342-345.

22 Romanick-Schmiedl S, Kiprov DD, Chalmers AC, Miller RG: Extraneural manifestations of chronic inflammatory demyelinating polyradiculoneuropathy. Am J Med 1990;89:531-534.

-23 Mobbs RJ, Tuck RR, Hurley B: Chronic inflammatory demyelinating polyneuropathy associated with membranous glomerulonephritis: case report. J Clin Neurosci 2000;7:454-455.

24 Froelich CJ, Searles RP, Davis LE, Goodwin JS: A case of Guillain-Barre syndrome with immunologic abnormalities. Ann Intern Med 1980;93:563-565.

25 Kitamura H, Nakano T, Kakihara M, et al: A case of Guillain-Barre syndrome developed minimal change nephrotic syndrome simultaneously. Am J Nephrol 1998;18:151-154.

-26 Qu Z, Liu G, Li J, et al: Absence of glomerular IgG4 deposition in patients with membranous nephropathy may indicate malignancy. Nephrol Dial Transplant 2012;27:1931-1937.

27 Prunotto M, Carnevali ML, Candiano G, et al: Autoimmunity in membranous nephropathy targets aldose reductase and SOD2. J Am Soc Nephrol 2010;21:507-519.

28 Bruschi M, Carnevali ML, Murtas C, et al: Direct characterization of target podocyte antigens and autoantibodies in human membranous glomerulonephritis: alfa-enolase and borderline antigens. J Proteomics 2011;74:2008-2017.

29 Aalberse RC, Stapel SO, Schuurman J, Rispens T: Immunoglobulin G4: An odd antibody. Clin Exp Allergy 2009;39:469-477.

30 Glassock RJ: The pathogenesis of idiopathic membranous nephropathy: a 50-year odyssey. Am J Kidney Dis 2010;56:157-167.

-31 Chiba A, Kusunoki S, Obata H, Machinami R, Kanazawa I: Serum anti-GQ1b IgG antibody is associated with ophthalmoplegia in Miller Fisher syndrome and Guillain-Barre syndrome: clinical and immunohistochemical studies. Neurology 1993;43:1911-1917. 


\section{Case Reports in \\ Nephrology and \\ Urology}

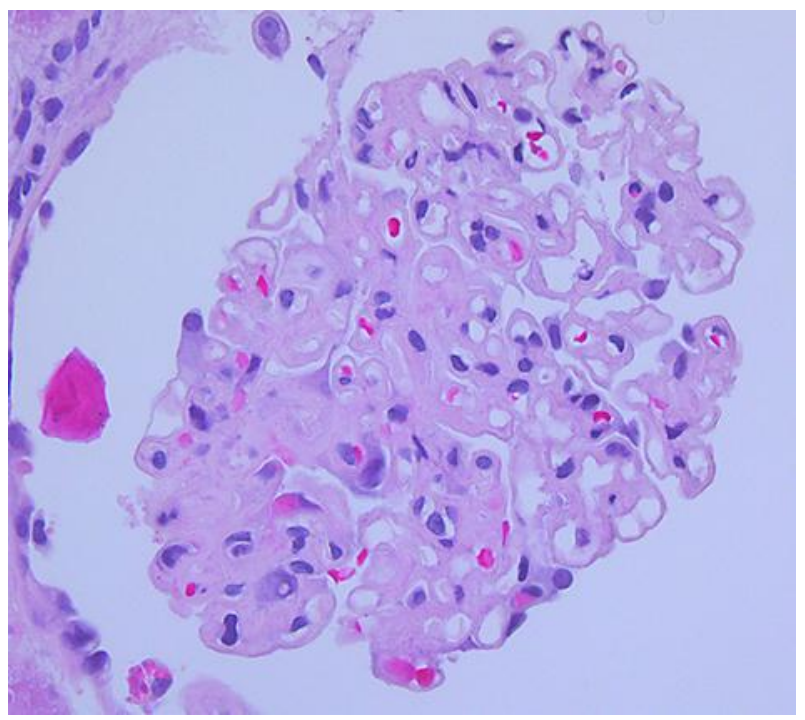

Fig. 1. Photomicrograph of a representative glomerulus from the renal biopsy. The glomerular basement membranes are thickened prominently and uniformly without evidence of cellular proliferation. Magnification, $\times 40$.

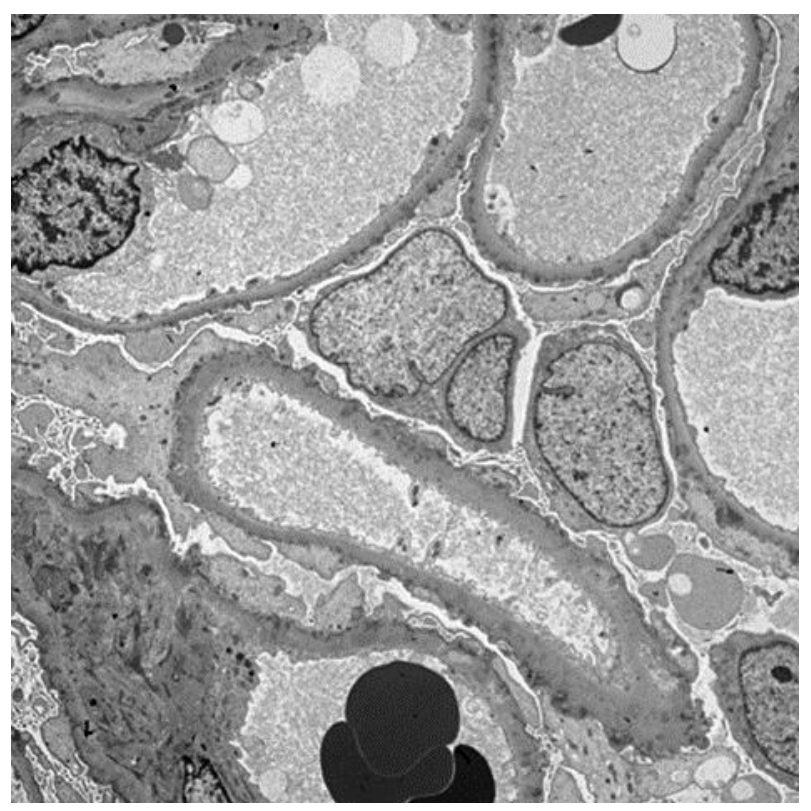

Fig. 2. Electron micrograph of a representative glomerulus from the renal biopsy. Subepithelial and intramembranous deposits are uniformly present throughout the glomerular basement membranes. Magnification, $\times 2,700$. 